\title{
The effect of the dexamethasone, cytarabine, and cisplatin (DHAP) regimen on stem cell mobili- zation and transplant outcomes of patients with non-Hodgkin's lymphoma who are candidates for up-front autologous stem cell transplantation
}

So Yeon Jeon ${ }^{1,2,}$, Ho-Young Yhim ${ }^{1,2,}$, , Hee Sun Kim ${ }^{3}$, Jeong-A Kim ${ }^{4}$, Deok-Hwan Yang ${ }^{5}$, and Jae-Yong Kwak ${ }^{1,2}$

\begin{abstract}
${ }^{1}$ Department of Internal Medicine, Chonbuk National University Medical School, Jeonju; ${ }^{2}$ Research Institute of Clinical Medicine of Chonbuk National University and Biomedical Research Institute of Chonbuk National University Hospital, Jeonju; ${ }^{3}$ Chonbuk National University College of Nursing, Jeonju; ${ }^{4}$ Division of Hematology, Department of Internal Medicine, College of Medicine, The Catholic University of Korea, Seoul; ${ }^{5}$ Department of Hematology-Oncology, Chonnam National University Hwasun Hospital, Hwasun, Korea
\end{abstract}

Received: May 1, 2016

Revised : September 1, 2016

Accepted: February 4, 2017

\section{Correspondence to}

Jae-Yong Kwak, M.D.

Department of Internal Medicine, Chonbuk National University Medical School, 20 Geonji-ro, Deokjin-gu, Jeonju 54907, Korea

Tel: +82-63-250-1791

Fax: +82-63-254-1609

E-mail:jykwak@jbnu.ac.kr

*These authors contributed equally to this work.
Background/Aims: Data on dexamethasone, cytarabine, and cisplatin (DHAP) as a mobilization regimen, compared to high-dose cyclophosphamide (HDC), for up-front autologous stem cell transplantation (ASCT) in non-Hodgkin's lymphoma (NHL) is limited.

Methods: Consecutive patients with aggressive NHL treated with cyclophosphamide, doxorubicin, vincristine, and prednisone (CHOP) or rituximab-CHOP who underwent chemomobilization using HDC or DHAP plus granulocyte-colony stimulating factor (G-CSF) for up-front ASCT were enrolled from three institutions between 2004 and 2014.

Results: Ninety-six patients (57 men) were included. Sixty-five patients (67.7\%) received HDC; and 31 (32.3\%), DHAP. The total $\mathrm{CD}_{34+}$ cells mobilized were significantly higher in patients receiving DHAP $\left(16.1 \mathrm{vs.} 6.1 \times 10^{6} / \mathrm{kg}, p=0.001\right)$. More patients achieved successful mobilization with DHAP $\left(\mathrm{CD}_{34}+\right.$ cells $\geq 5.0$ $\times 10^{6} / \mathrm{kg}$ ) compared to $\operatorname{HDC}(87.1 \%$ vs. $61.5 \%$, respectively; $p=0.011)$, particularly within the first two sessions of apheresis ( $64.5 \%$ vs. $32.3 \%$, respectively; $p=0.003$ ). Mobilization failure rate $\left(\mathrm{CD}_{34}+\right.$ cells $\left.<2.0 \times 10^{6} / \mathrm{kg}\right)$ was significantly higher in patients receiving $\mathrm{HDC}(20.0 \%$ vs. $3.2 \%, p=0.032)$. On multivariate analysis, the DHAP regimen (odds ratio, 4.12; 95\% confidence interval, 1.12 to 15.17) was an independent predictor of successful mobilization. During chemomobilization, patients receiving HDC experienced more episodes of febrile neutropenia compared to patients receiving DHAP (32.3\% vs. $12.9 \%, p=0.043$ ).

Conclusions: The DHAP regimen was associated with a significantly higher efficacy for stem cell mobilization and lower frequency of febrile neutropenia. Therefore, DHAP plus G-CSF is an effective for mobilization in patients with aggressive NHL who were candidates for up-front ASCT.

Keywords: Cyclophosphamide; Dexamethasone, cytarabine, and cisplatin; Mobilization; Lymphoma, non-Hodgkin; Autologous stem cell transplantation 


\section{INTRODUCTION}

High-dose chemotherapy with autologous stem cell transplantation (ASCT) remains an important treatment option for patients with non-Hodgkin's lymphoma (NHL) [1-3]. For successful ASCT, it is an essential first step to collect a sufficient amount of mobilized peripheral blood stem cells (PBSCs) [4]. However, inadequate PBSC mobilization may lead to treatment delay, potentially rendering some patients ineligible for planned ASCT $[5,6]$. In general, mobilization using chemotherapy combined with regular granulocyte-colony stimulating factor (G-CSF) stimulation is known to be associated with a higher mobilizing efficacy compared to G-CSF alone at some addition of costs and toxicities [7-10]. One of the common chemotherapeutic regimens for PBSC mobilization includes high-dose cyclophosphamide (HDC), with well-established efficacy of mobilization in various diseases and situations [7-13]. In addition, the dexamethasone, cytarabine, and cisplatin (DHAP) regimen has also been commonly used for chemomobilization of patients with lymphoma, and it was shown to be effective for PBSC mobilization [13-15]. However, these studies included a substantial number of lymphoma patients with relapsed or primary refractory diseases, who were previously exposed to various chemotherapy regimens [10]. These characteristics may greatly influence the comparison of the mobilizing efficacies of these chemotherapy regimens. Therefore, it is necessary to compare the mobilizing efficacy of these chemotherapy regimens in patients who are responsive to cyclophosphamide, doxorubicin, vincristine, and prednisone (CHOP) or rituximab-CHOP (R-CHOP) chemotherapy and candidates for up-front ASCT. However, until now, no specific data comparing the mobilizing efficacy and safety of the DHAP regimen with that of HDC as a chemomobilizer has been reported in this group of patients.

Currently, NHL is a heterogeneous disease with variable clinical behavior, response to therapy, and longterm outcomes [16-18]. Although treatment outcomes of aggressive NHL were remarkably improved in the recent year, approximately $40 \%$ of patients with diffuse large B-cell lymphoma (DLBCL) and $64 \%$ to $82 \%$ of patients with peripheral T-cell lymphomas (PTCLs) will eventually experience treatment failure within 5 years $[17,18]$. The fundamental benefit of chemomobilization is not only to improve mobilization efficacy but also to reduce graft contamination by malignant cells [19]. However, for tumor control, cyclophosphamide plus G-CSF is not theoretically preferred for mobilization in patients with lymphoma after cyclophosphamide-containing frontline chemotherapy such as CHOP or R-CHOP. The DHAP regimen is a disease-specific protocol, with significant anti-lymphoma activity $[20,21]$. Therefore, the disease-specific DHAP regimen for chemomobilization may provide additional tumor cytoreduction that could be translated into better clinical outcomes in aggressive NHL patients who undergo ASCT [19]. The aims of this study were to compare the efficacy of PBSC mobilization of the DHAP regimen with conventional HDC in combination with G-CSF in patients with aggressive NHL who were candidates for up-front ASCT, and to investigate the effect of DHAP chemomobilization on overall survival (OS).

\section{METHODS}

\section{Patient cohort}

We conducted a multicenter, retrospective cohort study on patients who were diagnosed with aggressive NHL and treated with CHOP or R-CHOP chemotherapy and subsequent PBSC mobilization using HDC or DHAP regimens for up-front ASCT in three South Korean academic hospitals between January 2004 and December 2014. Diagnosis of all included patients was established by tumor tissue biopsies obtained from the site of disease and reviewed by pathologist at each institution. Because this study would investigate the impact of mobilization regimen on OS as well as mobilization efficacy, patients were eligible if the histologic types were PTCL not otherwise specified, anaplastic lymphoma kinase-negative anaplastic large cell lymphoma, angioimmunoblastic T-cell lymphoma, and DLBCL with at least a partial response to CHOP or R-CHOP chemotherapy. Patients with DLBCL were only eligible if they were $\geq 2$ of age-adjusted International Prognostic Index (aaIPI) or bulky disease at baseline. Patients with lymphoblastic, Burkitt's, transformed, follicular (grade 3), mantle-cell, and other histologic type lymphomas were excluded from this analysis. We also excluded patients who had been treated with chemotherapy other than 
CHOP or R-CHOP before PBSC mobilization to reduce the confounding effects of prior chemotherapy on stem cell mobilization, and had primary or secondary central nervous system involvement at diagnosis. Baseline staging procedures included medical history, physical examination, complete blood counts, serum biochemistry with lactate dehydrogenase (LDH), computed tomography scanning of the chest, abdomen and pelvis, and bone marrow (BM) trephination biopsy. The study protocol was reviewed and approved by the Institutional Review Board (IRB, CUH 2014-04-004) at each institution, and the IRBs granted a waiver of informed consent because this study was a retrospective analysis that involved no more than minimal risk for patients.

\section{Autologous PBSC mobilization and collection}

PBSC was mobilized with either HDC or DHAP regimen plus G-CSF. The HDC was the intravenous administration of cyclophosphamide $\left(4.0 \mathrm{~g} / \mathrm{m}^{2}\right.$ on day 1$)$, and the DHAP regimen consisted of cisplatin $\left(100 \mathrm{mg} / \mathrm{m}^{2}\right.$ on day 1), cytarabine ( $4.0 \mathrm{~g} / \mathrm{m}^{2}$ on day 2 ), and dexamethasone (40 mg on day 1 to 4 ), intravenously. G-CSF (10 $\mu \mathrm{g} / \mathrm{kg} /$ day) was subcutaneously administered the day after the completion of chemotherapy or development of neutropenia (absolute neutrophil count $<1.0 \times 10^{3} / \mu \mathrm{L}$ ), as appropriate, until the final day of apheresis. After chemotherapy for PBSC mobilization, the first day of apheresis was determined by the monitoring of white blood cell (WBC) count or peripheral blood $\mathrm{CD}_{34+}$ cell count, which was initiated at the anticipated day of mobilization. In addition, we also included the hematopoietic progenitor cell (HPC) count, which was a small population of immature myeloid cells in the peripheral blood and could be identified by Sysmex automated hematology analyzer (Sysmex, Kobe, Japan), as a surrogate for time the initiation of collection procedure, because the number of circulating HPCs correlated with the yield of $\mathrm{CD}_{34}+$ cells [22]. PBSC collection usually began on the day when the peripheral blood $\mathrm{CD}_{34}+$ cells exceeded $10 / \mu \mathrm{L}$ or $\mathrm{HPC}$ counts exceeded $5 / \mu \mathrm{L}$. If peripheral blood $\mathrm{CD}_{34}+$ cell or HPC counts could not be used to determine the first day of apheresis, a WBC count of $>1.0 \times 10^{3} / \mu \mathrm{L}$ was usually considered as the best time point to start apheresis. Each apheresis procedure was performed according to each institution's protocol; however, most apheresis procedures were performed for approximately 3 to 4 hours, processing 10 to $14 \mathrm{~L}$ of blood volume. The collected total $\mathrm{CD}_{34+}$ cell counts were determined by flow cytometry from samples obtained before cryopreservation of apheresis products. Once the apheresis session was initiated, it was repeated daily until a sufficient number of CD34+ cells were collected.

For this analysis, we defined successful mobilization when collected $\mathrm{CD}_{34}+$ cells $>5.0 \times 10^{6} / \mathrm{kg}$, and optimal mobilization when collected $\mathrm{CD}_{34}+$ cells $\geq 2.0 \times 10^{6} / \mathrm{kg}$, whereas mobilization failure was defined by collected $\mathrm{CD}_{34}+$ cells $<2.0 \times 10^{6} / \mathrm{kg}$.

\section{High-dose chemotherapy with ASCT procedure}

The conditioning chemotherapy used consisted of busulfan/cyclophosphamide/etoposide, busulfan/melphalan/ etoposide, and BCNU/etoposide/cytarabine/melphalan. G-CSF was administered daily from 2 days after PBSC reinfusion until absolute neutrophil counts reached 1.0 $\times 10^{3} / \mu \mathrm{L}$ on 2 consecutive days. Supportive care such as transfusion and infection prophylaxis was administered during ASCT procedures in accordance with the institution's policy.

\section{Statistical analysis}

The primary end-points were the total number of collected $\mathrm{CD}_{34+}$ cells after chemomobilization, and OS. Clinical variables, such as age, sex, histologic type, Ann Arbor stage, presence of B symptoms, Eastern Cooperative Oncology Group (ECOG) performance status, LDH, bulky disease, aaIPI, BM involvement, primary chemotherapeutic regimen, number of chemotherapy cycles administered, disease status before PBSC mobilization, chemomobilization regimen, radiotherapy status, and information on the conditioning regimen were collected for analysis. Treatment response was assessed based on the previously reported criteria [23]. Descriptive statistics were presented as frequencies and percentages for categorical variables and as median and range for continuous variables. The two groups were compared according to chemomobilization regimen by Pearson chi-square test or Fisher exact test for categorical variables, and the Mann-Whitney test for continuous variables. To determine the optimal cutoff level for WBC counts at the first apheresis day on predicting successful mobilization, receiver operating characteristic (ROC) curve analysis was used. ROC curve analysis demon- 
strated that $1.785 \times 10^{3} / \mu \mathrm{L}$ had the best sensitivity (71.6\%) and specificity $(73.4 \%)$ to predict successful mobilization and the area under curve value was 0.732 . Multivariate analysis was performed using logistic regression model to find the prognostic factors associated with successful mobilization. Variables with significance level < 0.10 in univariate analyses were selected and included in the multivariate analysis, and the results were reported as an odds ratio (OR) and $95 \%$ confidence interval (CI).

Neutrophil engraftment is defined as the first date of 3 consecutive days with a neutrophil count $\geq 0.5 \times 10^{3} /$ $\mu \mathrm{L}$ and platelet engraftment as the first date of 7 consecutive days of platelet count $\geq 20 \times 10^{3} / \mu \mathrm{L}$ without platelet transfusion after PBSC reinfusion. Progression-free survival (PFS) and OS were calculated from the date of diagnosis until progression, death, or last follow-up, as appropriate. Time to neutrophil/platelet engraftments, PFS, and OS were estimated using the Kaplan-Meier method, and the differences between two groups were tested using a log-rank test. Cox proportional hazards regression model was used for univariate and multivariate comparison to find the prognostic factors for OS. Infused $\mathrm{CD}_{34}+$ cell counts of 33.3 percentile $\left(4.4 \times 10^{6}\right)$ $\mathrm{kg})$ and 66.7 percentile $\left(9.0 \times 10^{6} / \mathrm{kg}\right)$ had been initially regarded as cut-off points, but then 66.7 percentile (9.0 $\times 10^{6} / \mathrm{kg}$ ) was finally selected as a cut-off for binary comparison. A two-sided $p<0.05$ was considered statistically significant. All data analyses were carried out using SPSS software version 18.o (SPSS Inc., Chicago, IL, USA).

\section{RESULTS}

\section{Patient cohort and characteristics}

Ninety-six patients who satisfied the eligibility criteria were included in this analysis. The clinical characteristics of the enrolled patients at time of diagnosis are described in Table 1. Thirty-one patients received DHAP regimen and 65 patients received HDC regimen as mobilization chemotherapy. The most common histologic type was DLBCL ( $\mathrm{n}=52,54.2 \%)$; most of them ( $\mathrm{n}=$ 48) were initially treated with R-CHOP chemotherapy. Forty-seven patients (49\%) were Ann Arbor stage IV and 68 patients (70.8\%) had elevated serum LDH level at the time of diagnosis. Therefore, 63 patients $(65.6 \%)$ had high-intermediate or high aaIPI. Although the number of patients with extranodal disease was higher in HDC group compared to the DHAP group, premobilization characteristics were not significantly different between two groups (Table 1).

\section{Outcomes of PBSC mobilization and prognostic factors for successful mobilization}

Details of PBSC mobilization and collection according to mobilization chemotherapy are summarized in Table 2. The median number of total $\mathrm{CD}_{34}+$ cells collected per patient was significantly higher in the DHAP group than in the HDC group $\left(16.1 \times 10^{6} / \mathrm{kg}\right.$ vs. $6.1 \times 10^{6} / \mathrm{kg}, p$ $=0.001)$. In addition, the DHAP group had significantly higher number of $\mathrm{CD}_{34}+$ cells collected on apheresis days 1 and 2 than the HDC group (median, $8.25 \times 10^{6} /$ kg vs. $\left.2.97 \times 10^{6} / \mathrm{kg}, p=0.011\right)$. Thus, more patients in the DHAP group achieved successful mobilization compared to the HDC group (87.1\% vs. 61.5\%, $p=0.011)$, particularly within first two sessions of apheresis $(64.5 \%$ vs. $32.3 \%, p=0.003)$. The rate of mobilization failure was lower in the DHAP group than in the HDC group (3.2\% vs. $20.0 \%, p=0.032$ ), and consequently further attempt for chemomobilization was less frequent in the DHAP group compared to the HDC group (9.7\% vs. 33.8\%, $p=$ 0.012). Although there were no treatment-related mortality and grade 4 infections during chemomobilization, the HDC group experienced more episodes of febrile neutropenia compared to the DHAP group (32.3\% vs. 12.9\%, $p=0.043$ ) (Table 2).

In the univariate analysis, chemotherapy cycles administered $\leq 6$ cycles, WBC counts at first apheresis day $>1.785 \times 10^{3} / \mu \mathrm{L}$, and DHAP regimen were significant factors for successful mobilization (Table 3). No baseline BM involvement and no previous radiotherapy showed marginally significant trends for successful mobilization (Table 3). However, based on the multivariate analysis that included all these factors, no baseline BM involvement (OR, 4.60; 95\% CI, 1.20 to 17.61 ), no previous radiotherapy (OR, 11.78; 95\% CI, 1.68 to 82.39), WBC counts at first apheresis day $>1.785 \times 10^{3} / \mu \mathrm{L}$ (OR, 9.25; 95\% CI, 2.89 to 29.58), and the DHAP regimen (OR, 4.12; 95\% CI, 1.12 to 15.17 ) were independent predictors for successful mobilization (Table 3).

\section{ASCT outcomes and survival}

Of the 96 patients underwent PBSC mobilization and 
Table 1. Clinical characteristics of 96 patients according to mobilization regimens

\begin{tabular}{|c|c|c|c|c|}
\hline Characteristic & $\operatorname{Total}(n=96)$ & $\operatorname{DHAP}(\mathrm{n}=31)$ & $\operatorname{HDC}(\mathrm{n}=65)$ & $p$ value \\
\hline Age, yr & $47.5(18-66)$ & $49.0(24-66)$ & $47.0(18-65)$ & 0.337 \\
\hline Sex & & & & 0.532 \\
\hline Male & $57(59.4)$ & $17(54.8)$ & $40(61.5)$ & \\
\hline Female & $39(40.6)$ & $14(45.2)$ & $25(38.5)$ & \\
\hline Histologic types & & & & 0.729 \\
\hline DLBCL & $52(54.2)$ & $16(51.6)$ & $36(55.4)$ & \\
\hline PTCL & $44(45.8)$ & $15(48.4)$ & $29(44.6)$ & \\
\hline PTCL-NOS & 27 & 8 & 19 & \\
\hline ALCL, ALK(-) & 12 & 4 & 1 & \\
\hline AITL & 5 & 3 & 9 & \\
\hline Ann Arbor stage & & & & 0.342 \\
\hline I to III & $49(51.0)$ & $18(58.1)$ & $31(47.7)$ & \\
\hline IV & $47(49.0)$ & $13(41.9)$ & $34(52.3)$ & \\
\hline ECOG performance status & & & & 0.934 \\
\hline $0-1$ & $84(87.5)$ & $27(87.1)$ & $58(87.7)$ & \\
\hline $2-4$ & $12(12.5)$ & $4(12.9)$ & $8(12.3)$ & \\
\hline B symptoms & & & & 0.934 \\
\hline Absent & $66(68.8)$ & $27(87.1)$ & $58(87.7)$ & \\
\hline Present & $30(31.3)$ & $4(12.9)$ & $8(12.3)$ & \\
\hline LDH level & & & & 0.144 \\
\hline Normal & $28(29.2)$ & $6(19.4)$ & $22(33.8)$ & \\
\hline Elevated & $68(70.8)$ & $25(80.6)$ & $43(66.2)$ & \\
\hline Bulky disease & & & & 0.941 \\
\hline No & $78(81.2)$ & $25(80.6)$ & $53(80.0)$ & \\
\hline Yes & $18(18.8)$ & $6(19.4)$ & $13(20.0)$ & \\
\hline aaIPI & & & & 0.447 \\
\hline Low to low-intermediate & $33(34.4)$ & $9(29.0)$ & $24(36.9)$ & \\
\hline High-intermediate to high & $63(65.6)$ & $22(71.0)$ & $41(63.1)$ & \\
\hline BM involvement & & & & 0.780 \\
\hline No & $79(82.3)$ & $26(83.9)$ & $53(81.5)$ & \\
\hline Yes & $17(17.7)$ & $5(16.1)$ & $12(18.5)$ & \\
\hline No. of extranodal disease & $1(0-4)$ & $1(0-3)$ & $2(0-4)$ & 0.007 \\
\hline Primary chemotherapy & & & & 0.275 \\
\hline $\mathrm{R}-\mathrm{CHOP}$ & $48(50.0)$ & $18(58.1)$ & $30(46.2)$ & \\
\hline CHOP & $48(50.0)$ & $13(41.9)$ & $35(53.8)$ & \\
\hline No. of cycles administered & $6(4-8)$ & $6(4-8)$ & $6(4-8)$ & 0.101 \\
\hline Disease status before chemomobilization & & & & 0.380 \\
\hline CR & $86(89.6)$ & $29(93.5)$ & $57(87.7)$ & \\
\hline PR & $10(10.4)$ & $2(6.5)$ & $8(12.3)$ & \\
\hline Previous radiotherapy & & & & 0.645 \\
\hline No & $88(91.7)$ & $29(93.5)$ & $59(90.8)$ & \\
\hline Yes & $8(8.3)$ & $2(6.5)$ & $6(9.2)$ & \\
\hline
\end{tabular}

Values are presented as median (range) or number (\%).

DHAP, dexamethasone, cytarabine, and cisplatin; HDC, high-dose cyclophosphamide; DLBCL, diffuse large B-cell lymphoma; PTCL, peripheral T-cell lymphoma; NOS, not otherwise specified; ALCL, anaplastic large cell lymphoma; ALK, anaplastic lymphoma kinase; AITL, angioimmunoblastic T-cell lymphoma; ECOG, Eastern Cooperative Oncology Group; LDH, lactate dehydrogenase; aaIPI, age-adjusted International Prognostic Index; BM, bone marrow; R, rituximab; CHOP, cyclophosphamide, doxorubicin, vincristine, and prednisone; CR, complete response; PR, partial response. 
Table 2. Outcomes of PBSC mobilization according to chemotherapy regimens

\begin{tabular}{|c|c|c|c|c|}
\hline Variable & $\operatorname{Total}(\mathrm{n}=96)$ & $\operatorname{DHAP}(\mathrm{n}=31)$ & $\operatorname{HDC}(\mathrm{n}=65)$ & $p$ value \\
\hline Days from chemotherapy to first apheresis & $13(10-19)$ & $12(10-15)$ & $13(11-19)$ & $<0.001$ \\
\hline WBC counts at first apheresis day, $\times 10^{3} / \mu \mathrm{L}$ & $2.085(0.590-23.810)$ & $3.300(0.590-23.810)$ & $1.910(0.830-13.420)$ & 0.134 \\
\hline No. of apheresis & $4(2-7)$ & $4(3-7)$ & $4(2-7)$ & 0.128 \\
\hline Total CD $34+$ cells collected, $\times 10^{6} / \mathrm{kg}$ & $10.4(0.2-98.9)$ & $16.1(1.9-38.8)$ & $6.1(0.2-98.9)$ & 0.001 \\
\hline $\mathrm{CD}_{34}+$ cells collected at days $1-2, \times 10^{6} / \mathrm{kg}$ & $4.32(0.09 c 62.18)$ & $8.25(0.30-18.91)$ & $2.97(0.09-62.18)$ & 0.011 \\
\hline Successful mobilization, $\geq 5 \times 10^{6} / \mathrm{kg}$ & $67(69.8)$ & $27(87.1)$ & $40(61.5)$ & 0.011 \\
\hline Successful mobilization at day $1-2$ & $41(42.7)$ & $20(64.5)$ & $21(32.3)$ & 0.003 \\
\hline Optimal mobilization, $\geq 2 \times 10^{6} / \mathrm{kg}$ & $82(85.4)$ & $30(96.8)$ & $52(80.0)$ & 0.032 \\
\hline Mobilization failure, $<2 \times 10^{6} / \mathrm{kg}$ & $14(14.6)$ & $1(3.2)$ & $13(20.0)$ & 0.032 \\
\hline Further attempt for chemomobilization & $25(26.0)$ & $3(9.7)$ & $22(33.8)$ & 0.012 \\
\hline Febrile neutropenia & $25(26.0)$ & $4(12.9)$ & $21(32.3)$ & 0.043 \\
\hline Treatment-related mortality & 0 & o & 0 & - \\
\hline
\end{tabular}

Values are presented as median (range) or number (\%).

PBSC, peripheral blood stem cell; DHAP, dexamethasone, cytarabine, and cisplatin; HDC, high-dose cyclophosphamide; WBC, white blood cell.

collection, two patients did not proceed to up-front ASCT because of failed mobilization in one patient (HDC group) and patient's refusal in one (DHAP group). All 94 patients underwent up-front ASCT received busulfan-based conditioning chemotherapy (Table 4). Although higher number of $\mathrm{CD}_{34}+$ cells were infused in the DHAP group compared to the HDC group $\left(8.4 \times 10^{6}\right)$ kg vs. $6.0 \times 10^{6} / \mathrm{kg}, p=0.021$ ) during ASCT procedures, time to neutrophil engraftment (median, 11 days vs. 11 days, $p=0.310$ ) and platelet engraftment (median, 12 days vs. 14 days, $p=0.876$ ) were not different according to chemomobilization regimen (Table 4).

With a median follow-up of 57.4 months (range, 9.1 to 143.4), the 5-year PFS rate was 66.2\% (95\% CI, 55.2 to 77.2) and the 5-year OS rate was $72.9 \%$ (95\% CI, 62.5 to 83.3). In the DHAP group, the 5 -year PFS and OS rates were 63.2\% (95\% CI, 44.4 to 82.0 ) and $74.0 \%$ (95\% CI, 56.8 to 91.2), and the 5 -year PFS and OS rates in the HDC group were $67.6 \%$ (95\% CI, 54.1 to 81.1) and $72.2 \%$ (95\% CI, 59.3 to 85.1), respectively, which showed no significant differences between the two groups $(p>0.05)$ (Fig. $1 \mathrm{~A}$ and $1 D)$. Because of the heterogeneous histologic types of patients included in this study cohort, additional subgroup analyses were performed separately based on the histologic types. However, in both DLBCL (Fig. 1B and ${ }_{1} \mathrm{E}$ ) and PTCL subgroups (Fig. $1 \mathrm{C}$ and ${ }_{1 \mathrm{~F}}$ ), the 5 -year
PFS (DLBCL, $59.1 \%$ vs. 57.5\%, $p=0.878$; PTCL, $64.0 \%$ vs. $74.3 \%, p=0.611$ ) and OS (DLBCL, $81.3 \%$ vs. $68.0 \%, p=$ 0.776; PTCL, $70.9 \%$ vs. $76.3 \%, p=0.812$ ) were not different between the DHAP and the HDC groups. When we compared the PFS and OS according to infused $\mathrm{CD}_{34}+$ cell dose, patients who received $>9.0 \times 10^{6} / \mathrm{kg}$ showed significantly better 5-year PFS (89.1\% vs. $55.8 \%, p=0.008$ ) and $\mathrm{OS}(90.8 \%$ vs. $65.4 \%, p=0.009)$ than those with $\leq 9.0$ $\times 10^{6} / \mathrm{kg}$ (Fig. 2).

The results of the univariate and multivariate Cox regression analyses for OS are shown in Table 5. The univariate analysis suggested that Ann Arbor stage IV, ECOG performance status $\geq 2$, elevated serum LDH level, high-intermediate/high aaIPI risk, and infused $\mathrm{CD}_{34+}$ cell counts were potential prognostic indicators for OS. However, multivariate analysis that included these factors revealed that only infused $\mathrm{CD}_{34}+$ cell counts $>9.0 \times$ $10^{6} / \mathrm{kg}$ was an independent prognostic factor for better OS (hazard ratio, 0.42; 95\% CI, 0.20 to 0.87).

Among 10 patients with partial response before chemomobilization, six patients (two in DHAP group and four in HDC group) evaluated the response to mobilization chemotherapy. Of these, responses were upgraded into complete response after chemomobilization in each one patient from both DHAP and HDC groups. However, because of too small fraction of these patients, 
Table 3. Prognostic factors for successful mobilization

\begin{tabular}{|c|c|c|c|c|c|c|}
\hline \multirow{2}{*}{ Variable } & \multirow{2}{*}{ No. } & \multirow{2}{*}{$\begin{array}{l}\text { The rate of successful } \\
\text { mobilization, \% (95\% CI) }\end{array}$} & \multicolumn{2}{|c|}{ Univariate analysis } & \multicolumn{2}{|c|}{ Multivariate analysis ${ }^{\mathrm{a}}$} \\
\hline & & & OR $(95 \% \mathrm{CI})$ & $p$ value & OR $(95 \% \mathrm{CI})$ & $p$ value \\
\hline \multicolumn{7}{|l|}{ Age, yr } \\
\hline$\geq 48$ & 48 & $70.8(49.1-99.0)$ & 1 & & & \\
\hline$<48$ & 48 & $68.8(47 \cdot 3-96.6)$ & $0.91(0.38-2.17)$ & 0.824 & & \\
\hline \multicolumn{7}{|l|}{ Sex } \\
\hline Male & 57 & $73.7(53.1-99.6)$ & 1 & & & \\
\hline Female & 39 & $64.1(41.5-94.6)$ & $0.64(0.26-1.54)$ & 0.317 & & \\
\hline \multicolumn{7}{|l|}{ Histologic types } \\
\hline DLBCL & 52 & $67.3(46.9-93.6)$ & 1 & & & \\
\hline PTCL & 44 & $72.7(49.7-100.0)$ & $1.30(0.54-3.13)$ & 0.565 & & \\
\hline \multicolumn{7}{|l|}{ Ann Arbor stage } \\
\hline I to III & 49 & $73.5(51.5-100.0)$ & 1 & & & \\
\hline IV & 47 & $66.0(44.8-93.6)$ & $0.70(0.29-1.68)$ & 0.424 & & \\
\hline \multicolumn{7}{|l|}{ LDH level } \\
\hline Normal & 28 & $71.4(43.6-100.0)$ & 1 & & & \\
\hline Elevated & 68 & $78.3(57.6-100.0)$ & $1.12(0.42-2.94)$ & 0.823 & & \\
\hline \multicolumn{7}{|l|}{ aaIPI } \\
\hline Low to LI & 48 & $69.7(44.2-100.0)$ & 1 & & & \\
\hline HI to high & 47 & $69.8(50.7-93.8)$ & $1.01(0.40-2.52)$ & 0.988 & & \\
\hline \multicolumn{7}{|c|}{ Baseline BM involvement } \\
\hline Yes & 17 & $52.9(24.2-100.0)$ & 1 & & 1 & \\
\hline No & 79 & $73.4(55.7-94.9)$ & $2.46(0.84-7.20)$ & 0.098 & $4.60(1.20-17.61)$ & 0.026 \\
\hline \multicolumn{7}{|c|}{ Disease status before chemo-mobilization } \\
\hline $\mathrm{PR}$ & 10 & $50.0(16.2-100.0)$ & 1 & & & \\
\hline CR & 86 & $72.1(55.3-92.4)$ & $2.58(0.69-9.73)$ & 0.161 & & \\
\hline \multicolumn{7}{|c|}{ Chemotherapy cycles } \\
\hline$>6$ & 25 & $52.0(27.7-88.9)$ & 1 & & & \\
\hline$\leq 6$ & 71 & $76.1(57.1-99.2)$ & $2.93(1.13-7.62)$ & 0.027 & NS & NS \\
\hline \multicolumn{7}{|c|}{ Previous radiotherapy } \\
\hline Yes & 8 & $37.5(7 \cdot 7-10.0)$ & 1 & & & \\
\hline No & 88 & $72.7(56.0-92.9)$ & $4.44(0.99-20.04)$ & 0.052 & $11.78(1.68-82.39)$ & 0.013 \\
\hline \multicolumn{7}{|c|}{ WBC counts at first apheresis day } \\
\hline$\leq 1.785 \times 10^{3} / \mu \mathrm{L}$ & 40 & $47.5(28.6-74.2)$ & 1 & & & \\
\hline$>1.785 \times 10^{3} / \mu \mathrm{L}$ & 56 & $85.7(63.2-100.0)$ & $6.63\left(2.5^{1-17.53)}\right.$ & $<0.001$ & $9.25(2.89-29.58)$ & $<0.001$ \\
\hline \multicolumn{7}{|c|}{ Mobilization regimen } \\
\hline $\mathrm{HDC}$ & 65 & $61.5(44.0-83.8)$ & 1 & & 1 & \\
\hline DHAP & 31 & $87.1(57.4-100.0)$ & $4.22(1.32-13.50)$ & 0.015 & $4.12(1.12-15.17)$ & 0.033 \\
\hline
\end{tabular}

CI, confidence interval; OR, odds ratio; DLBCL, diffuse large B-cell lymphoma; PTCL, peripheral T-cell lymphoma; LDH, lactate dehydrogenase; aaIPI, age-adjusted International Prognostic Index; LI, low-intermediate; HI, high-intermediate; BM, bone marrow; PR, partial response; CR, complete response; NS, not significant; WBC, white blood cell; HDC, high-dose cyclophosphamide; DHAP, dexamethasone, cytarabine, and cisplatin.

adjusted by baseline BM involvement, number of chemotherapy cycles, previous radiotherapy, total WBC counts at first apheresis day, and mobilization regimen. 
Table 4. Outcomes of autologous stem cell transplantation

\begin{tabular}{|c|c|c|c|c|}
\hline Variable & Total $(\mathrm{n}=94)$ & $\operatorname{DHAP}(\mathrm{n}=30)$ & $\operatorname{HDC}(\mathrm{n}=64)$ & $p$ value \\
\hline Conditioning regimen & & & & 0.001 \\
\hline Busulfan/cyclophosphamide/etoposide & $55(58.5)$ & $9(30.0)$ & $46(71.9)$ & \\
\hline Busulfan/etoposide/cytarabine/melphalan & $20(21.3)$ & $10(33 \cdot 3)$ & $10(15.6)$ & \\
\hline Busulfan/melphalan/etoposide & $19(20.2)$ & $11(36.7)$ & $8(12.5)$ & \\
\hline Infused $\mathrm{CD}_{34}+$ cells, $\times 10^{6} / \mathrm{kg}$ & $6.4(1.7-50.5)$ & $8.4(2.1-29.0)$ & $6.0(1.7-50.5)$ & 0.021 \\
\hline Time to neutrophil engraftment, day & $11(8-26)$ & $11(9-26)$ & $11(8-25)$ & 0.310 \\
\hline Time to platelet engraftment, day & $13(7-60)$ & $12(8-60)$ & $14(7-43)$ & 0.876 \\
\hline Treatment-related mortality & $4(4 \cdot 3)$ & $1(3 \cdot 3)$ & $3(4 \cdot 7)$ & 1.000 \\
\hline
\end{tabular}

Values are presented as number (\%) or median (range).

DHAP, dexamethasone, cytarabine, and cisplatin; HDC, high-dose cyclophosphamide.
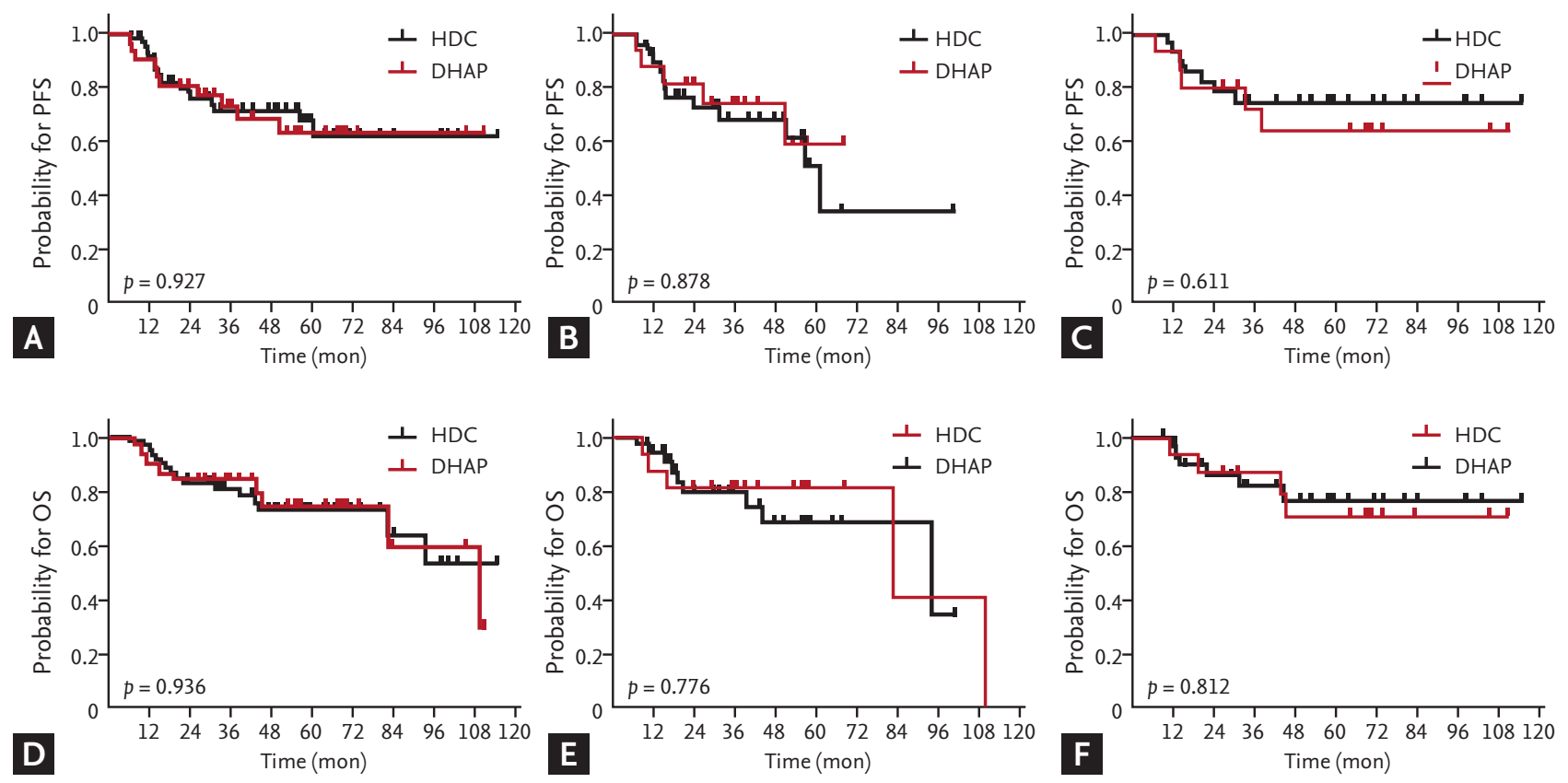

Figure 1. Comparison of survival outcomes according to the mobilization chemotherapy regimen. In all patients included in the study cohort, (A) progression-free survival (PFS) and (D) overall survival (OS) were not different between patients who received dexamethasone, cytarabine, and cisplatin (DHAP) or high-dose cyclophosphamide (HDC). Furthermore, in the subgroup analysis, the mobilization chemotherapy regimen, DHAP or HDC, did not significantly affect PFS and OS in patients with diffuse large B-cell lymphoma (B: PFS; E, OS) or peripheral T-cell lymphoma (C: PFS; F: OS).

we couldn't evaluate the additional cytoreductive effect of DHAP regimen.

\section{DISCUSSION}

In this multicenter, retrospective cohort study involving 96 NHL patients, we investigated the effects of DHAP plus G-CSF as a mobilization regimen on efficacy of PBSC mobilization, incidence of febrile neutropenia, and PFS and OS, compared to HDC plus G-CSF in patients with NHL, who were responsive to front-line CHOP or R-CHOP chemotherapy. Our study demonstrated that the DHAP regimen plus G-CSF was superior to HDC plus G-CSF in terms of total collected CD34+ cell counts, $\mathrm{CD}_{34}+$ cells collected on days 1 to 2 , and successful mo- 

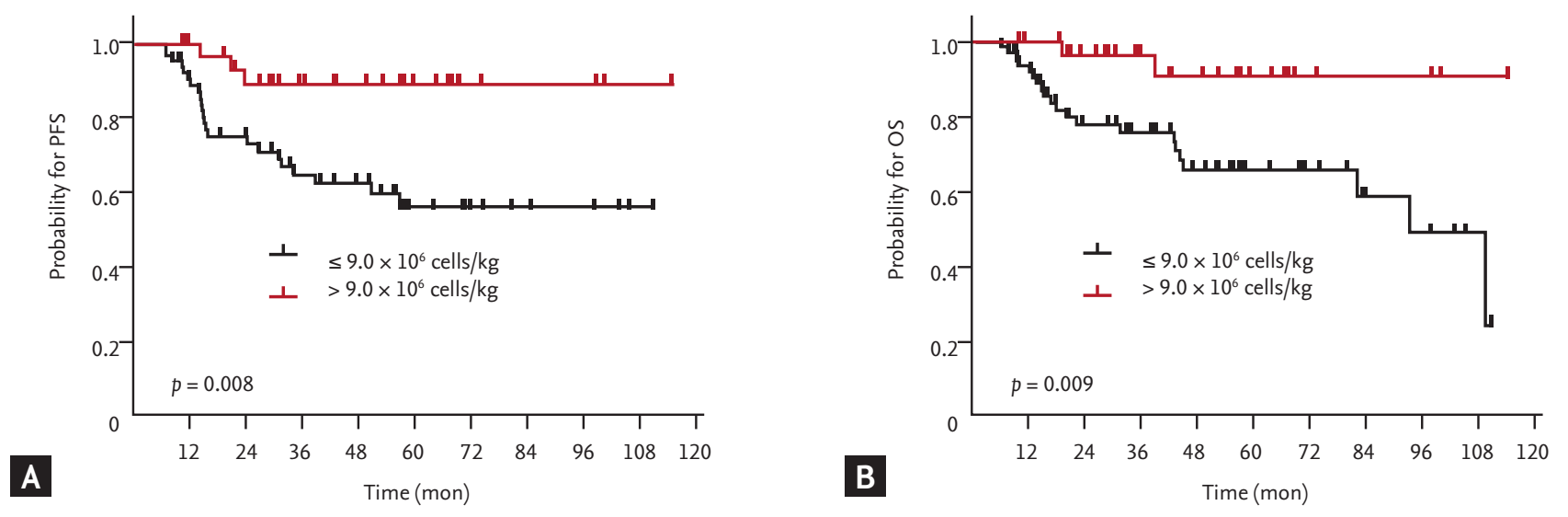

Figure 2. Survival outcomes according to infused CD $34+$ cell counts. Patients infused with $\mathrm{CD}_{34}+\mathrm{cells}>9.0 \times 10^{6} / \mathrm{kg}$ produced significantly longer (A) progression-free survival (PFS) and (B) overall survival (OS) compared to those with CD34+ cells $\leq 9.0 \times$ $10^{6} / \mathrm{kg}$.

bilization. However, the disease-specific DHAP regimen did not influence the PFS and OS in the present analysis. The DHAP regimen was also associated with lower rate of febrile neutropenia and mobilization failure than the HDC regimen. Moreover, the DHAP regimen was an independent predictor for successful mobilization. Therefore, our results suggested that the DHAP regimen might be a suitable option for chemomobilization in patients with aggressive NHL. However, the results of the present study cannot be extrapolated to all settings of PBSC mobilization in patients with NHL. The findings observed in this study were slightly different from the results of previous study. Pavone et al. [14] previously reported that the DHAP regimen was as effective as, but not superior to the HDC with regard to the outcomes of mobilizing efficacy. We think that the discrepancy of the results between two studies may be mainly attributed to the differences in patient population. In the study of Pavone et al. [14], they included significant proportion of patients with primary refractory/relapsed disease and prior exposure of DHAP regimen, whereas our study only included patients with chemo-responsive disease, who were treated with CHOP or R-CHOP regimens and could be candidates for up-front ASCT. Notably, it has been well known that PBSC mobilization efficacy was greatly influenced by underlying disease status and previous exposure to chemotherapeutic agents such as platinum compounds $[10,15,19,24]$. Indeed, because of negative effect of salvage chemotherapy and primary re- fractory/relapsed disease status on mobilization efficacy, it is inadequate to compare the mobilizing efficacy of chemomobilization regimens directly in these groups of patients. In this sense, our study may provide the evidence for the positive effect of the DHAP regimen on mobilizing efficacy in patients with NHL for up-front ASCT. Moreover, it has been generally considered that chemomobilization could mobilize well in only patients able to have a potential to mobilize well [4], because previous data suggested that chemomobilization may mobilize more stem cells than G-CSF alone but with a similar rate of failure $[7,9,12]$. However, in this study, the DHAP regimen had significantly lower rates of mobilization failure than the HDC regimen. This finding suggested that the DHAP regimen might save some patients who would experience mobilization failure if they had received the HDC regimen. In addition, although grade 4 infections were not observed in both groups, incidence of febrile neutropenia was significantly higher in the HDC group (32\%), which was consistent with the results of previous studies $[13,25,26]$. Therefore, considering the mobilizing efficacy and safety of the DHAP regimen observed in this study, the DHAP regimen can be considered as reference chemomobilization regimen for up-front ASCT in patients with aggressive NHL.

Chemotherapy during PBSC mobilization may lead to further tumor cytoreduction and in vivo purging of mobilized tumor cells [19]. Furthermore, intensive chemomobilization of PBSC in patients with NHL is more 
Table 5. Univariate and multivariate Cox proportional hazards regression analyses for OS

\begin{tabular}{|c|c|c|c|c|}
\hline \multirow{2}{*}{ Variable } & \multicolumn{2}{|c|}{ Univariate analysis } & \multicolumn{2}{|c|}{ Multivariate analysis } \\
\hline & $\operatorname{HR}(95 \% \mathrm{CI})$ & $p$ value & $\operatorname{HR}(95 \% \mathrm{CI})$ & $p$ value \\
\hline \multicolumn{5}{|l|}{ Age, yr } \\
\hline$<60$ & 1 & & & \\
\hline$\geq 60$ & $1.77(0.41-7.58)$ & 0.443 & & \\
\hline \multicolumn{5}{|l|}{ Sex } \\
\hline Male & 1 & & & \\
\hline Female & $0.83(0.35-1.95)$ & 0.661 & & \\
\hline \multicolumn{5}{|l|}{ Ann Arbor stage } \\
\hline I to III & 1 & & & \\
\hline IV & $2.20(0.98-4.93)$ & 0.053 & NS & NS \\
\hline \multicolumn{5}{|l|}{ B symptoms } \\
\hline Absent & 1 & & & \\
\hline Present & $1.09(0.46-2.54)$ & 0.850 & & \\
\hline \multicolumn{5}{|c|}{ ECOG performance status } \\
\hline $0-1$ & 1 & & & \\
\hline $2-4$ & $2.51(0.54-11.63)$ & 0.078 & NS & NS \\
\hline \multicolumn{5}{|l|}{ LDH level } \\
\hline Normal & 1 & & & \\
\hline Elevated & $2.47(1.06-5.78)$ & 0.086 & NS & NS \\
\hline \multicolumn{5}{|l|}{ aaIPI } \\
\hline Low to LI & 1 & & & \\
\hline HI to high & $3.13(1.39-7.13)$ & 0.027 & NS & NS \\
\hline \multicolumn{5}{|l|}{ BM involvement } \\
\hline No & 1 & & & \\
\hline Yes & $1.83(0.67-5.03)$ & 0.239 & & \\
\hline \multicolumn{5}{|l|}{ Histologic type } \\
\hline DLBCL & 1 & & & \\
\hline PTCL & $0.61(0.27-1.38)$ & 0.233 & & \\
\hline \multicolumn{5}{|c|}{ Disease status before mobilization } \\
\hline CR & 1 & & & \\
\hline PR & $1.50(0.44-5.09)$ & 0.518 & & \\
\hline \multicolumn{5}{|c|}{ Mobilization regimen } \\
\hline DHAP & 1 & & & \\
\hline HDC & $1.04\left(0.45^{-2.38)}\right.$ & 0.936 & & \\
\hline \multicolumn{5}{|c|}{ Infused CD $34+$ cells } \\
\hline$\leq 9.0 \times 10^{6} / \mathrm{kg}$ & 1 & & & \\
\hline$>9.0 \times 10^{6} / \mathrm{kg}$ & $0.18(0.08-0.42)$ & 0.008 & $0.42(0.20-0.87)$ & 0.020 \\
\hline
\end{tabular}

OS, overall survival; HR, hazards ratio; CI, confidence interval; NS, not significant; ECOG, Eastern Cooperative Oncology Group; LDH, lactate dehydrogenase; aaIPI, age-adjusted International Prognostic Index; LI, low-intermediate; HI, high-intermediate; BM, bone marrow; DLBCL, diffuse large B-cell lymphoma; PTCL, peripheral T-cell lymphoma; CR, complete response; PR, partial response; DHAP, dexamethasone, cytarabine, and cisplatin; HDC, high-dose cyclophosphamide. 
efficient than non-intensive mobilization [19,27]. Thus, it was hypothesized that the disease-specific chemotherapeutic regimens used for PBSC collection might not only facilitate PBSC mobilization but also have an anti-tumor effect that can be translated into improved survival outcomes. However, in the present study, disease-specific intensive DHAP regimen did not improve the PFS and OS compared to the conventional HDC regimen. This finding implied that anti-tumor effect of the DHAP regimen for PBSC mobilization was minimal or clinically irrelevant. Absolutely, our study included only patients who were candidates for up-front ASCT, and thus survival benefit from chemotherapy for mobilization may be smaller than expected in the salvage ASCT. Therefore, we cannot exclude the possibility that the small number of patients included may affect these results. Nevertheless, our findings suggest that anti-tumor effects of mobilization chemotherapy do not need to be preferentially considered for up-front ASCT.

Interestingly, our analysis demonstrated that higher infused $\mathrm{CD}_{34}+$ cell count was an independent predictor of better OS regardless of the chemomobilization regimens used, which was in agreement with the findings of previous reports [28-30]. Infusion of higher $\mathrm{CD}_{34}+$ cells may lead to early engraft and possibly protect against post-transplant infectious complications [31]. This may be related to the survival benefit of higher dose $\mathrm{CD}_{34+}$ cell infusion. However, because the treatment-related mortality was generally less than $5 \%$ in ASCT, it is unlikely that the benefit of higher $\mathrm{CD}_{34}+$ cell infusion was solely due to less infectious complications from early engraftment. Instead, several previous reports suggested that infusion of higher $\mathrm{CD}_{34}+$ cells might be associated with early recovery of immunologic activity against tumor cells, and consequent eradication of minimal residual disease, which leading to better clinical outcomes [28-30,32]. Until now, it has not been known what the exact immunologic mechanisms are and how higher $\mathrm{CD}_{34}+$ cells work to eradicate the minimal residual disease. However, because higher $\mathrm{CD}_{34}+$ cell mobilization and collection is the prerequisite for higher $\mathrm{CD}_{34}+$ cell infusion, patients infused higher $\mathrm{CD}_{34}+$ cells may have various favorable clinical factors for better PBSC mobilization [30]. It has been well known that more healthier BM and better disease status before mobilization were associated with higher $\mathrm{CD}_{34+}$ cell mobilization
$[10,15,19,24]$. In the multivariate analysis for successful chemomobilization in the present study, no baseline $\mathrm{BM}$ involvement and no prior radiotherapy were also independent factors for successful mobilization. Thus, although we did not exactly know the relationship between higher $\mathrm{CD}_{34+}$ cell infusion and improved survival, these findings suggested that infusion of higher CD34+ cells might act as a surrogate marker for better clinical factors or other as-yet-known laboratory factors. Furthermore, the optimal dose of $\mathrm{CD}_{34}+$ cells for improved survival has not yet been defined. Therefore, the influence of $\mathrm{CD}_{34+}+$ cell dose on immunologic recovery and meaningful OS improvement requires further investigation.

Our study has several limitations. First, the present analysis is a retrospective study with a small number of patients, so unexpected selection bias may exist. Second, the results of preapheresis circulating peripheral blood $\mathrm{CD}_{34}+$ cell counts, which was identified as a valuable factor for predicting efficacy of PBSC mobilization [9,19], was not included in the multivariate analysis, because data was available for only 25 patients (26\%). Third, mobilization with G-CSF alone in patients with lymphoma was not a routine policy in three institutions included in this study because lymphoma had been traditionally considered as difficult-to-mobilize disease [4,19]. Moreover, until now, plerixafor is not approved for up-front mobilization or preemptive use in Korea. Therefore, the effect of G-CSF alone or G-CSF plus plerixafor as first-line mobilizer on efficacy of PBSC mobilization was beyond the scope of the present analysis. However, considering the fact that PBSC mobilization with G-CSF alone was comparable to cyclophosphamide plus G-CSF in NHL patients who were not heavily pretreated [10], we believed that comparative studies between chemomobilization plus G-CSF and G-CSF alone would be needed in this group of patients. Nevertheless, our study is the first to show that the DHAP regimen had better stem cell mobilizing efficacy than the HDC regimen in patients with aggressive NHL, who were candidates for upfront ASCT.

In conclusion, our study showed that the DHAP regimen was associated with higher efficacy for PBSC mobilization, in particular early days of apheresis, and less frequent episodes of febrile neutropenia compared to the HDC regimen. Although there were no clinically 
meaningful PFS and OS improvement in patients mobilized with DHAP regimen, the DHAP regimen reduced significant rates of mobilization failure. Therefore, the results of this study may allow physicians to select a chemomobilization regimen for up-front ASCT in patients with aggressive NHL. Furthermore, the relevance of high $\mathrm{CD}_{34}+$ cell dose infusion should also be further explored because new effective drugs were under development for PBSC mobilization.

\section{KEY MESSAGE}

1. Limited data are available regarding the optimal chemomobilization regimen for patients with aggressive non-Hodgkin's lymphoma (NHL).

2. Compared to high-dose cyclophosphamide chemomobilization, our results demonstrate that the dexamethasone, cytarabine, and cisplatin (DHAP) regimen has better mobilizing efficacy and safety for up-front autologous stem cell transplantation in patients with aggressive NHL.

\section{Conflict of interest}

No potential conflict of interest relevant to this article was reported.

\section{REFERENCES}

1. Stiff PJ, Unger JM, Cook JR, et al. Autologous transplantation as consolidation for aggressive non-Hodgkin's lymphoma. N Engl J Med 2013;369:1681-1690.

2. Villanueva ML, Vose JM. The role of hematopoietic stem cell transplantation in non-Hodgkin lymphoma. Clin Adv Hematol Oncol 2006;4:521-530.

3. Lee HG, Choi Y, Kim SY, et al. R-CHOP chemoimmunotherapy followed by autologous transplantation for the treatment of diffuse large B-cell lymphoma. Blood Res 2014;49:107-114.

4. Giralt S, Costa L, Schriber J, et al. Optimizing autologous stem cell mobilization strategies to improve patient outcomes: consensus guidelines and recommendations. Biol Blood Marrow Transplant 2014;20:295-308.

5. Sanchez-Ortega I, Querol S, Encuentra M, et al. Plerixafor in patients with lymphoma and multiple myeloma: effectiveness in cases with very low circulating $\mathrm{CD}_{34+}$ cell levels and preemptive intervention vs remobilization. Bone Marrow Transplant 2015;50:34-39.

6. Wuchter P, Ran D, Bruckner T, et al. Poor mobilization of hematopoietic stem cells-definitions, incidence, risk factors, and impact on outcome of autologous transplantation. Biol Blood Marrow Transplant 2010;16:490-499.

7. Sung AD, Grima DT, Bernard LM, et al. Outcomes and costs of autologous stem cell mobilization with chemotherapy plus G-CSF vs G-CSF alone. Bone Marrow Transplant 2013;48:1444-1449.

8. Narayanasami U, Kanteti R, Morelli J, et al. Randomized trial of filgrastim versus chemotherapy and filgrastim mobilization of hematopoietic progenitor cells for rescue in autologous transplantation. Blood 2001;98:2059-2064.

9. Pusic I, Jiang SY, Landua S, et al. Impact of mobilization and remobilization strategies on achieving sufficient stem cell yields for autologous transplantation. Biol Blood Marrow Transplant 2008;14:1045-1056.

10. Milone G, Leotta S, Indelicato F, et al. G-CSF alone vs cyclophosphamide plus G-CSF in PBPC mobilization of patients with lymphoma: results depend on degree of previous pretreatment. Bone Marrow Transplant 2003;31:747-754.

11. Dazzi C, Cariello A, Rosti G, et al. Is there any difference in PBPC mobilization between cyclophosphamide plus G-CSF and G-CSF alone in patients with non-Hodgkin's Lymphoma? Leuk Lymphoma 2000;39:301-310.

12. Dingli D, Nowakowski GS, Dispenzieri A, et al. Cyclophosphamide mobilization does not improve outcome in patients receiving stem cell transplantation for multiple myeloma. Clin Lymphoma Myeloma 2006;6:384-388.

13. Hyun SY, Cheong JW, Kim SJ, et al. High-dose etoposide plus granulocyte colony-stimulating factor as an effective chemomobilization regimen for autologous stem cell transplantation in patients with non-Hodgkin lymphoma previously treated with CHOP-based chemotherapy: a study from the Consortium for Improving Survival of Lymphoma. Biol Blood Marrow Transplant 2014;20:73-79.

14. Pavone V, Gaudio F, Guarini A, et al. Mobilization of peripheral blood stem cells with high-dose cyclophosphamide or the DHAP regimen plus G-CSF in non-Hodgkin's lymphoma. Bone Marrow Transplant 2002;29:285-290.

15. Pavone V, Gaudio F, Console G, et al. Poor mobilization is an independent prognostic factor in patients with ma- 
lignant lymphomas treated by peripheral blood stem cell transplantation. Bone Marrow Transplant 2006;37:719-724.

16. Kwak JY. Treatment of diffuse large B cell lymphoma. Korean J Intern Med 2012;27:369-377.

17. Sehn LH, Gascoyne RD. Diffuse large B-cell lymphoma: optimizing outcome in the context of clinical and biologic heterogeneity. Blood 2015;125:22-32.

18. Vose J, Armitage J, Weisenburger D; International T-Cell Lymphoma Project. International peripheral T-cell and natural killer/T-cell lymphoma study: pathology findings and clinical outcomes. J Clin Oncol 2008;26:4124-4130.

19. Gertz MA. Current status of stem cell mobilization. Br J Haematol 2010;150:647-662.

20. Gisselbrecht C, Schmitz N, Mounier N, et al. Rituximab maintenance therapy after autologous stem-cell transplantation in patients with relapsed $\mathrm{CD} 2 \mathrm{O}(+)$ diffuse large B-cell lymphoma: final analysis of the collaborative trial in relapsed aggressive lymphoma. J Clin Oncol 2012;30:4462-4469.

21. Philip T, Guglielmi C, Hagenbeek A, et al. Autologous bone marrow transplantation as compared with salvage chemotherapy in relapses of chemotherapy-sensitive non-Hodgkin's lymphoma. N Engl J Med 1995;333:15401545 .

22. Suh C, Kim S, Kim SH, et al. Initiation of peripheral blood progenitor cell harvest based on peripheral blood hematopoietic progenitor cell counts enumerated by the Sysmex SE9000. Transfusion 2004;44:1762-1768.

23. Cheson BD, Pfistner B, Juweid ME, et al. Revised response criteria for malignant lymphoma. J Clin Oncol 2007;25:579-586.

24. Ford CD, Green W, Warenski S, Petersen FB. Effect of prior chemotherapy on hematopoietic stem cell mobilization. Bone Marrow Transplant 2004;33:901-905.
25. Jillella AP, Ustun C, Robach E, et al. Infectious complications in patients receiving mobilization chemotherapy for autologous peripheral blood stem cell collection. J Hematother Stem Cell Res 2003;12:155-160.

26. Ahn JS, Park S, Im SA, et al. High-dose versus low-dose cyclophosphamide in combination with G-CSF for peripheral blood progenitor cell mobilization. Korean J Intern Med 2005;20:224-231.

27. Damon L, Damon LE, Gaensler K, et al. Impact of intensive PBSC mobilization therapy on outcomes following auto-SCT for non-Hodgkin's lymphoma. Bone Marrow Transplant 2008;42:649-657.

28. Yoon DH, Sohn BS, Jang G, et al. Higher infused CD34+ hematopoietic stem cell dose correlates with earlier lymphocyte recovery and better clinical outcome after autologous stem cell transplantation in non-Hodgkin's lymphoma. Transfusion 2009;49:1890-1900.

29. Gordan LN, Sugrue MW, Lynch JW, Williams KD, Khan SA, Moreb JS. Correlation of early lymphocyte recovery and progression-free survival after autologous stem-cell transplant in patients with Hodgkin's and non-Hodgkin's Lymphoma. Bone Marrow Transplant 2003;31:1009-1013.

30. Sauter CS, Giralt S. The prognostic impact of peripheral blood progenitor cell dose following high-dose therapy and autologous stem cell transplant for hematologic malignancies. Leuk Lymphoma 2015;56:1619-1625.

31. Ketterer N, Salles G, Raba M, et al. High CD34(+) cell counts decrease hematologic toxicity of autologous peripheral blood progenitor cell transplantation. Blood 1998;91:3148-3155.

32. Porrata LF, Gertz MA, Inwards DJ, et al. Early lymphocyte recovery predicts superior survival after autologous hematopoietic stem cell transplantation in multiple myeloma or non-Hodgkin lymphoma. Blood 2001;98:579-585 